\title{
Reflets
}

Revue d'intervention sociale et communautaire

C. Amaratunga, J. Gahagan (dir.), Striking to the Heart of the Matter : Selected Readings on Gender and HIV, Halifax, Atlantic Centre of Excellence for Women's Health, 2002, 106 p.

C. Amaratunga (dir.), Race, Ethnicity and Women's Health, Halifax, Atlantic Centre of Excellence for Women's Health, 2002, 206 p.

\section{Jacinthe Michaud}

Volume 9, numéro 1, printemps 2003

Le genre en contexte : pratiques sociales et représentations

URI : https://id.erudit.org/iderudit/010874ar

DOI : https://doi.org/10.7202/010874ar

Aller au sommaire du numéro

Éditeur(s)

Reflets : Revue ontaroise d'intervention sociale et communautaire

ISSN

1203-4576 (imprimé)

1712-8498 (numérique)

Découvrir la revue

Citer ce compte rendu

Michaud, J. (2003). Compte rendu de [C. Amaratunga, J. Gahagan (dir.), Striking to the Heart of the Matter : Selected Readings on Gender and HIV, Halifax,

Atlantic Centre of Excellence for Women's Health, 2002, 106 p. / C. Amaratunga (dir.), Race, Ethnicity and Women's Health, Halifax, Atlantic Centre of Excellence for Women's Health, 2002, 206 p.] Reflets, 9(1), 269-274. https://doi.org/10.7202/010874ar

Tous droits réservés (C) Reflets : Revue ontaroise d'intervention sociale et communautaire, 2002
Ce document est protégé par la loi sur le droit d'auteur. L'utilisation des services d'Érudit (y compris la reproduction) est assujettie à sa politique d'utilisation que vous pouvez consulter en ligne.

https://apropos.erudit.org/fr/usagers/politique-dutilisation/ 


\section{Striking to the Heart of the Matter: Selected Readings on Gender and HIV et \\ Race, Ethnicity and women's Health}

\section{Carol Amaratunga et Jacqueline Gahagan (dir.)}

Striking to the Heart of the Matter: Selected Readings on Gender and HIV, Halifax: Atlantic Centre of Excellence for Women's Health, 2002, 106 pages.

Carol Amaratunga (dir.)

Race, Ethnicity and Women's Health, Halifax: Atlantic Centre of Excellence for Women's Health, 2002, 206 pages.

Le Centre d'excellence sur la santé des femmes de l'Atlantique publiait l'an dernier deux ouvrages réunissant des articles, l'un sur le genre et le Sida, Striking to the Heart of the Matter: Selected Readings on Gender and HIV et l'autre sur la race, l'ethnicité et la santé des femmes, Race, Ethnicity and Women's Health. Ces deux publications s'inscrivent dans l'orientation du Centre d'excellence, à savoir, parvenir à combler le manque critique de connaissances sur la santé des femmes des provinces maritimes, en particulier des femmes issues de groupes socialement et économiquement désavantagés. Elles cherchent également à faire la lumière sur les barrières qui limitent l'accès aux services de santé et les difficultés qu'éprouvent certaines catégories sociales de femmes à influencer 
l'orientation des politiques de santé. Striking to the Heart of the Matter et Race Ethnicity and Women's Health proposent une vision féministe sur des enjeux précis : la reconnaissance qu'au Canada, plus de femmes sont contaminées et meurent de Sida que les hommes ; que le racisme, la violence, la pauvreté et l'exclusion des services de santé ont une influence sur le taux d'infection ; que les méthodologies de recherche doivent s'intéresser au vécu et aux expériences des femmes à qui ces recherches prétendent s'adresser; que les services et l'orientation des politiques publiques en matière de santé et de services sociaux doivent prendre en considération les déterminants sociaux et économiques plutôt que de mettre l'accent sur les comportements individuels. Telles sont quelques-unes des orientations et des conclusions des recherches rassemblées dans ces deux ouvrages.

Striking to the Heart of the Matter, rassemble des articles ayant une perspective canadienne sur le Sida et les femmes. Le premier texte, "The Economic Cost of HIV/AIDS in Canada ", sert en quelque sorte d'introduction à l'ouvrage en faisant le point sur les coûts économiques et sociaux du VIH/SIDA. La prévalence de la maladie, en particulier du côté des jeunes femmes de moins de 30 ans, autochtones pauvres et utilisatrices de drogues, devrait, selon les auteur-es, forcer les experts et les décideurs politiques à reposer la question à partir d'une approche multidisciplinaire. Dans la mesure où le profil des personnes infectées - jusqu'ici organisé autour du modèle gai et masculin qui a influencé l'orientation des politiques et des services - s'est modifié au cours de la dernière décennie, il devient de plus en plus important d'adopter une approche inspirée des rapports sociaux de sexe dans l'analyse du phénomène. Dans cet article, Colin Dodds et al., présentent de nombreuses statistiques sur la progression du VIH/SIDA au Canada, les types de personnes affectées, l'incidence de l'hétérosexualité dans le nombre de tests positifs et les coûts associés au traitement de la maladie.

À ce premier exposé de la situation statistique et économique, s'ajoute un deuxième chapitre sur les enjeux soulevés par les méthodes de recherche à l'endroit des groupes sociaux exclus. Margreth Tolson and Stephanie Kellington estiment que la 
recherche conventionnelle en sciences sociales fait entendre la voix de certains groupes de personnes à l'exclusion d'autres types de parole, soit la voix des experts qui s'expriment au détriment d'une clientèle déjà victimisée en vertu de leur appartenance à certains groupes d'individus ou associée à certains comportements jugés pathologiques. Ces modèles traditionnels de recherche tendent à poser des jugements individualistes et moralisateurs plutôt que de mettre l'accent sur les déterminants de la santé. Les auteures proposent donc un tout autre modèle de recherche dont elles ont pu observer le fonctionnement auprès de groupes de femmes séropositives de Vancouver. Il s'agit d'une méthode de recherche participante appelée "Listen UP ! Research Project ". Plusieurs constats se dégagent de cette expérience, entre autres, qu'il y a des liens entre des situations de violence et le VIH : violence institutionnelle, violence de la rue, violence dans les relations intimes.

Ces violences multivariées et qui se superposent, s'imposeront comme autant de constats aux lectrices et aux lecteurs au fil des réalités marginalisées exposées dans des contextes différenciés. Aussi, n'est-il plus possible de faire abstraction des effets à long terme de la colonisation sur les femmes autochtones, colonisation toujours pratiquée à leur endroit et qui les rend plus vulnérables à une infection au HIV. Les femmes autochtones et inuits venues du Nord se retrouvent dans les grandes villes telles que Montréal, Toronto etVancouver, en raison de l'anonymat que leur procurent ces grands complexes urbains. Ces femmes, qui au sein de leur communauté d'origine étaient infirmières, enseignantes, travailleuses sociales, se retrouvent aujourd'hui à la merci de professionnels-les qui les victimisent. Un autre article à retenir est celui de Lois Jackson, "HIV Prevention Programme and Female Prostitutes : The Canadian Context ». Les prostituées, davantage que n'importe quelle autre catégorie de personnes, sont particulièrement au fait des dangers de leur profession et savent mieux que quiconque se protéger contre les infections. Faisant un usage généralisé du condom, le comportement sécuritaire d'un bon nombre de prostituées vient contredire les croyances qui les identifient comme les principales responsables 
de la transmission de la maladie. Ici encore la violence qu'elles subissent de la part de leurs clients doit être prise en considération dans l'organisation des services et l'orientation des politiques de santé. Plutôt que d'associer leur comportement à de la déviance et à de la pathologie, l'approche institutionnelle dans le traitement du VIH/SIDA chez les prostituées devrait plutôt interroger les déterminants sociaux auxquels elles sont soumises.

Contrairement au premier ouvrage, Race, Ethnicity and Women's Health se concentre essentiellement sur les provinces maritimes, surtout sur les populations noires, acadiennes et immigrantes. La directrice de publication de cette collection, Carol Amarantunga, estime que les obstacles contrevenant à l'accessibilité aux soins et aux services de santé sont de trois ordres : celui de la langue et de la communication, celui de la culture et celui de la recherche médicale. Il se dégage de la diversité des articles rassemblés tout un ensemble de préoccupations suivies de recommandations similaires à celles qui ont inspiré l'ouvrage précédent, c'est-à-dire reconnaitre qu'il est urgent de pallier le manque de connaissances sur la santé de certaines catégories sociales de femmes - vivant ici dans les provinces atlantiques - cela afin d'influencer les politiques publiques en matière de santé et de réorienter plus adéquatement l'organisation des services vers celles qui en ont été exclues jusqu'à présent.

La première étape serait de parvenir à une meilleure compréhension de la dynamique d'exclusion. Wanda Thomas Bernard s'y emploie dans "Beyond Inclusion ». Par exemple, par rapport à un des déterminants de la santé — l'éducation — l'auteure posera la question de la proportion des autochtones, des acadiennes, des immigrantes, des africaines canadiennes et des femmes handicapées parmi les diplômé-es post-secondaires. À propos de la redistribution des revenus et du mode de vie, elle fera valoir que $70 \%$ des mères seules vivent en dessous du seuil de pauvreté et que la majorité des enfants de la région vivent dans des familles à parent unique. Ces statistiques sont alarmantes ajoute-t-elle, surtout lorsqu'elles sont mises en relations avec d'autres facteurs. D'ailleurs, aucune analyse adéquate sur l'inclusion/exclusion des services de santé ne saurait être entreprise sans que ne soit prise en 
considération la dynamique d'attribution et de distribution du pouvoir et des privilèges.

Les auteures, Erica van Roosmalen, Charlotte Loppie, Karina Davidson dans "Women's Voices : Atlantic Canadian Women's Contribution to Health Policy " nous offrent un chapitre intéressant sur la perception et l'expérience que diverses catégories sociales de femmes ont de leur santé et des services qu'elles reçoivent. Le projet qu'elles ont réalisé auprès de ces femmes visait aussi la collaboration de plusieurs acteurs communautaires, universitaires et gouvernementaux : une sorte de recherche participante où des groupes d'acteurs font l'expérience du partenariat. Sur le plan méthodologique, plusieurs catégories sociales de femmes (autochtones, femmes noires, femmes de militaires, femmes francophones et acadiennes, etc) ont été entendues à l'intérieur de plusieurs groupes témoins. Dans la mesure où les chercheures s'inscrivaient dans une approche comparative, chacune des catégories de femmes a été considérée de façon autonome bien que les chercheures aient été conscientes de la diversité qui traverse la vie de chacune de ces femmes. Toute approche faisant ressortir la perception des femmes vis-à-vis leur propre santé et les services qu'elles reçoivent est toujours intéressante et celle-ci a eu le mérite d'illustrer à quel point les femmes savent qu'elles ont peu d'emprise sur les orientations des politiques publiques et l'organisation des services de santé et des services sociaux. Cependant, même s'il y a quelques extraits d'entrevues, nous aurions aimé en lire davantage; nous aurions aimé aussi une analyse plus approfondie du sexisme et de l'ethnocentrisme anglo-saxon, bien plus que simplement mentionner que ce fait pose un problème pour les femmes noires, autochtones, francophones et/ou acadiennes.

Striking to the Heart of the Matter: Selected Readings on Gender and HIV et Race Ethnicity and Women's Health s'ajoutent à la collection d'ouvrages ayant servi de point de départ à des analyses théoriques plus approfondies sur la santé des femmes et ses déterminants sociaux, économiques, politiques et culturels. Plusieurs articles offrent une bibliographie (articles et documents de recherche) très élaborée sur leur sujet respectif. Il s'agit 
d'ouvrages qui, sans aucun doute, seront utiles pour l'enseignement de cours de premier cycle en Études des femmes, en sociologie de la santé des femmes et en analyse féministe des politiques publiques.

\section{Jacinthe Michaud}

École d'Études des femmes, Université York 